\title{
LOS INDIGENISMOS LÉXICOS EN LA DESCRIPCIÓN GEOGRÁFICO-MORAL DEL ARZOBISPO CORTÉS Y LARRAZ (1770): LOS DATOS SALVADOREÑOS
}

José Luis Ramírez Luengo

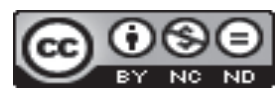

Doi: https://doi.org/10.15517/rfl.v45i2.39129

URL: https://revistas.ucr.ac.cr/index.php/filyling/index 



\title{
LOS INDIGENISMOS LÉXICOS EN LA DESCRIPCIÓN GEOGRÁFICO-MORAL DEL ARZOBISPO CORTÉS Y LARRAZ (1770): LOS DATOS SALVADOREÑOS
}

\author{
LEXICAL INDIGENISMS IN ARCHBISHOP CORTÉS Y \\ LARRAZ'S DESCRIPCIÓN GEOGRÁFICO-MORAL (1770): \\ SOME SALVADOREAN DATA
}

José Luis Ramírez Luengo

\begin{abstract}
RESUMEN
El presente trabajo se entiende como un intento de paliar, en parte, la escasez de estudios que existe sobre la historia léxica del español centroamericano. Con este propósito, se pretende analizar los indigenismos que se pueden descubrir en los capítulos que el arzobispo de Guatemala, Pedro Cortés y Larraz, dedica a El Salvador en su Descripción geográfico-moral de la diócesis de Goathemala (1770). De este modo, se tienen en cuenta cuestiones como el origen etimológico de las voces, su grado de integración en el español o los campos semánticos en que se incorporan, y se presta una especial atención a aquellas unidades que se pueden considerar americanismos desde una perspectiva de uso y dinámica de este concepto. Todo lo anterior tiene la intención final de detectar la presencia de elementos diatópicamente marcados y, por tanto, de subrayar el proceso de dialectalización de este nivel lingüístico que, por medio de los indigenismos, se puede descubrir ya en el español de la región en las postrimerías del siglo XVIII.

Palabras clave: Historia del español de América; El Salvador; siglo XVIII; léxico; indigenismo.
\end{abstract}

\begin{abstract}
This paper aims to be an attempt to decrease the lack of studies about the lexical history of Central American Spanish, so its main goal is to analyse Indigenous lexical items (indigenisms) that can be found in the chapters devoted to El Salvador in the Descripción geográfica-moral de la diócesis de Goathemala, a chronicle written in 1770 by Pedro Cortés y Larraz, archbishop of Guatemala. Thus, issues like etymological origin of indigenisms, their integration degree, semantic fields where they are used or items that can be considered as americanisms from a dynamic and usage point of view are taken into account. The final purpose of the analysis is to detect diatopically marked words and to describe the dialectalization of this linguistic level that, by means of indigenisms, can be discovered in this region at the end of $18^{\text {th }}$ century.

Keywords: History of Latin American Spanish; El Salvador; $18^{\text {th }}$ century; lexicon; indigenisms.
\end{abstract}

Dr. José Luis Ramírez Luengo. Profesor en la Facultad de Lenguas y Letras de la Universidad Autónoma de Quétaro. México.

Correo electrónico: joseluis.ramirezluengo@gmail.com

Recibido: 29- 11- 18

Aceptado: 8- 01- 19 


\section{La historia del español salvadoreño: un hueco en blanco ${ }^{1}$}

No constituye ninguna novedad -más bien todo lo contrario- referirse una vez más al alto grado de desconocimiento que afecta a la historia del español centroamericano, sin duda una de las áreas más abandonadas de todo el continente en lo que se refiere a esta cuestión. Si bien es verdad que en los últimos años se han publicado algunos trabajos acerca de esta temática ${ }^{2}$, lo cierto es que aún queda mucho por estudiar al respecto, y esto es especialmente evidente en casos como el de El Salvador, país sobre el que apenas existen unos pocos análisis muy parciales sobre la situación lingüística del siglo XVIII (Ramírez Luengo, 2010; en prensa; y en prensa b) y sobre el léxico decimonónico que aparece en la Memoria del estado político y eclesiástico de la capitanía general de Guatemala del santaneco Méndez y Cordero (Ramírez Luengo, 2017c y 2018). Así pues, no es exagerado afirmar que es prácticamente todo lo que aún falta por investigar antes de poder ofrecer una visión más o menos completa de los procesos diacrónicos que determinan la configuración del español que hoy se utiliza en el país. Se hace preciso, por tanto, realizar cuanto antes nuevos estudios que, tomando como base los diversos corpus disponibles actualmente ${ }^{3}$, permitan en un futuro no muy lejano aportar nuevos datos que vayan poco a poco reconstruyendo la desconocida historia del español salvadoreño y de sus variedades regionales.

\section{El trabajo, I: corpus y objetivos}

A partir del diagnóstico anterior, el presente trabajo pretende ser una contribución a la historia lingüística de El Salvador -y más en concreto, al estudio diacrónico de su léxico- por medio del análisis de los indigenismos que aparecen en la sección dedicada a este país en la Descripción geográfico-moral de la diócesis de Goathemala, extensísimo y detallado informe que redacta en 1770 Pedro Cortés y Larraz, arzobispo de Guatemala, a resultas de una visita pastoral de dos años (1768-1770) por los diversos territorios que componen su arquidiócesis ${ }^{4}$.

Tal y como señala Aguilar y Avilés (2000, pp. 9-10), Cortés y Larraz nace en Belchite (Zaragoza) en 1712, y desarrolla su vida en la capital aragonesa -primero como estudiante, después como canónigo de la catedral- hasta que, en 1766, es propuesto por Carlos III como

1 Este trabajo constituye uno de los productos resultantes del proyecto de investigación Léxico histórico del español de El Salvador tardocolonial (1650-1803) (FFL-2018-02), financiado por la Universidad Autónoma de Querétaro por medio del Fondo para el Fortalecimiento de la Investigación UAQ-2018.

2 Muy especialmente para la variedad costarricense, que es la única que cuenta por el momento con un trabajo completo que abarca toda su historia (Quesada Pacheco, 2009); a este se deben sumar otros estudios sobre aspectos puntuales desarrollados por este autor (Quesada Pacheco, 1987 y 2013) y por otros (León Fernández, 1988; Ulate Zúñiga, 1991).

3 Quizá uno de los problemas que determinaba la escasez de estudios diacrónicos fuera precisamente la falta de corpus sobre los que llevarlos a cabo; por suerte, también esto comienza a cambiar, tanto por la aparición de antologías documentales (Ruz, 2002-2004; Ramírez Luengo, 2017d) como por la publicación de obras de variada naturaleza -tales como la crónica utilizada para este trabajo (Cortés y Larraz, 2000)- que permiten ahora desarrollar una investigación prácticamente imposible hasta no hace demasiado tiempo.

4 Al igual que en Ramírez Luengo (en prensa b), en este estudio se emplea la edición parcial de la obra, centrada en las parroquias del actual territorio salvadoreño, que publica CONCULTURA en el año 2000 (Cortés y Larraz, 2000); téngase en cuenta, por tanto, que todos los ejemplos citados en el texto con la página de aparición remiten a esta edición concreta.

Doi: https://doi.org/10.15517/rfl.v45i2.39129 / URL: https://revistas.ucr.ac.cr/index.php/filyling/index 
arzobispo de Guatemala; como tal se traslada a la sede del arzobispado, adonde llega en 1768 para embarcarse inmediatamente en una vista pastoral de dos años por los territorios que hoy comprenden las repúblicas de Guatemala y El Salvador. Fruto de la visita es la Descripción geográfico-moral de la Diócesis de Goathemala que se va a estudiar en estas páginas (Aguilar y Avilés, 2000, p. 13). Posteriormente, como resultado de su negativa a trasladarse a la nueva capital del reino tras el terremoto de 1773, Cortés y Larraz se enfrenta a las autoridades civiles guatemaltecas y esto determina la intervención de la Corona y su vuelta a España como obispo de Tortosa, cargo que ocupa hasta 1786 cuando decide retirarse a Zaragoza, ciudad en la que fallece en julio de 1787 (Aguilar y Avilés, 2000, pp. 16-18) ${ }^{5}$.

Por lo que se refiere a la obra, la Descripción geográfico-moral se puede entender como un texto de naturaleza híbrida pero cercano a los informes ilustrados, en el que, a partir de un patrón constante ${ }^{6}$, se lleva a cabo una detallada exposición de las principales características físicas, económicas y sociales de cada una de las parroquias que componen el arzobispado. Tras esta descripción, se incorporan -por medio de la fórmula "y hasta aquí el cura"- las reflexiones personales de Cortés y Larraz sobre la situación de la parroquia, basadas en su observación in situ, las cuales suelen adquirir generalmente tintes bastante pesimistas (Aguilar y Avilés, 2000, pp. 13-15)7. De este modo, el hecho de que la obra se configure como una descripción minuciosa de la realidad salvadoreña de finales del siglo XVIII resalta su importancia para el estudio del léxico del español de esta región en el periodo indicado, pues cuestiones como "el interés del prelado por reflejar fielmente la realidad que observa en su visita" o "la amplitud de temas tratados en su escrito [...] que guardan relación con las costumbres propias de la zona” por fuerza van a determinar la aparición en el texto de "un vocabulario cercano a la cotidianeidad y, por tanto, susceptible de ofrecer las voces diatópicamente marcadas que caracterizan a esta variedad del español" (Ramírez Luengo, en prensa b) ${ }^{8}$.

En cuanto a la organización del estudio, este comenzará por señalar el listado de indigenismos presentes en el corpus para, posteriormente, examinar su grado de integración y las estrategias discursivas de incorporación textual de las voces no integradas. Tras este primer acercamiento, se llevará a cabo el análisis del origen etimológico de los vocablos

5 Para datos biográficos más detallados, véase la obra fundamental de Martín Blasco y García Añoveros (1992).

6 Tal patrón no es más que la transposición al texto del cuestionario que el propio prelado plantea en la carta pastoral que envía el 23 de septiembre de 1768 a los sacerdotes de su diócesis, y que consta de diez preguntas: 1) rentas de la parroquia; 2) idiomas hablados; 3) pueblos y caseríos de la comprensión; 4) familias y habitantes; 5) escándalos y abusos notados; 6) si los parroquianos cumplen con los principales preceptos; 7) si hay escuelas con maestros de calidad; 8) si han notado idolatrías y supersticiones; 9) cuáles libros se usan para explicar la doctrina; y 10) si los indios son castigados en exceso y los motivos para ello (Aguilar y Avilés, 2000, p. 10).

7 Para una descripción más precisa del contenido de la obra, véase Cortés y Larraz (2000, pp. 33-44); resulta también interesante el trabajo de García Añoveros (1980) para un análisis de la importancia que posee la Descripción para la historia social de la Guatemala dieciochesca.

8 A este respecto, el origen peninsular de Cortés y Larraz permite suponer que gran parte de los indigenismos que van a aparecer en el texto constituyen unidades léxicas que se incorporan a su variedad nativa de español como resultado de los procesos de contacto interdialectal experimentados durante su estancia en Guatemala. Lo anterior, a su vez, asegura el empleo de tales unidades léxicas, si no específicamente en El Salvador, al menos en la región centroamericana. Sobre los efectos que, en el campo del léxico, produce el contacto interdialectal, véase Ramírez Luengo (2014), así como la bibliografía citada en ese trabajo. 
registrados, su distribución en campos léxicos y su aparición como primeras dataciones, absolutas o centroamericanas, así como su carácter de americanismos en el siglo XVIII desde una perspectiva de uso y dinámica de este concepto (Company, 2007, pp. 28-29; Ramírez Luengo, 2015, p. 116). Por último, el trabajo se cierra con unas conclusiones en las que se pone de manifiesto la incidencia de estos elementos léxicos de origen amerindio en el proceso de dialectalización que, en el caso del español salvadoreño, afecta a este nivel lingüístico ya en la segunda mitad del Siglo Ilustrado? .

\section{El trabajo, II: los indigenismos en la Descripción de Cortés y Larraz}

Tal y como se señaló en otro estudio sobre esta cuestión (Ramírez Luengo, en prensa b), la Descripción geográfico-moral se caracteriza por presentar una riqueza léxica realmente notable, algo que se debe a circunstancias tan variadas como el alto nivel cultural de su autor, la tipología textual en la que se enclava la obra, su finalidad eminentemente informativa o la complejidad temática que muestra. Así las cosas, no sorprende que en sus páginas hagan acto de presencia cierta cantidad de indigenismos que son utilizados por Cortés y Larraz para referirse a diferentes realidades americanas y que -como se verá-constituyen una de las vetas léxicas más interesantes de su extenso informe.

De este modo, el expurgo de todos los capítulos salvadoreños de la Descripción ofrece 25 términos de origen autóctono, que son los siguientes: atole, bejuco, cacahuatal, cacao, cajete, calpul, canoa, ceiba, chicha, chile, chocolate, copal, jacal, jiquilite, maíz, macegual (mazahual $\left.{ }^{1 \sigma}\right)$, metate, milpa, nahual, nahuite, pajuide, petate, tapanco, tule, zompopero ${ }^{11}$. A estos se deben sumar, además, otros cuatro elementos: guayabal, joco, jocote, pitajaya, ya

9 Por supuesto, no son solo los indigenismos los que contribuyen a dotar de personalidad propia a la variedad de español que es empleada en la región en estos momentos, ni son los únicos vocablos diatópicamente restringidos que aparecen en la Descripción del arzobispo aragonés. Para una visión general de los americanismos -indígenas o endohispánicos- que aparecen en la sección salvadoreña de la obra, véase Ramírez Luengo (en prensa b).

10 Aunque no cabe duda de que el prelado se refiere al término macegual, cabe indicar que es esta la variante que aparece en el texto: "desdichados de los mazahuales, que es la gente ordinaria, si los indios fueran señores de la América” (Cortés y Larraz, 2000, p. 136). A este respecto, la aparición de un ejemplo de esta forma en López de Gómara en el siglo XVI ("assí dizen mazauali por labrador"; CORDE, 2016) descarta la opción de que el uso de Cortés y Larraz sea un mero error gráfico y avala la presencia de esta variante en la Guatemala del siglo XVIII, por más que tanto las obras lexicográficas (DLE, 2014, s.v. macegual; DAMER, 2010, s.v. macegual; DHECR, 1995, s.v. macegual; Herranz, 2017, s.v. macegual; Morínigo, 1998, s.v. macegual) como los corpus históricos consultados (CORDE, 2016; CORDIAM, 2016; LEXHISP, 2003) demuestren un claro predominio de macegual.

11 En concreto, la localización de los indigenismos en la edición utilizada (Cortés y Larraz, 2000) es la siguiente: atole (p. 129), bejuco (p. 206), cacahuatal (p. 77), cacao (pp. 70, 74, 77, 118), cajete (p. 115), calpul (p. 83), canoa (pp. 207, 2018), ceiba (p. 77), chicha (pp. 62, 63, 75, 198), chile (p. 136), chocolate (p. 83), copal (p. 76), jacal (pp. 62, 74, 80, 80, 90, 97, 98, 104, 106, 112, 114, 134, 136, 137, 139, 141, 144, $152,161,163,163,200,202$ ), jiquilite (p. 166), maiz (pp. 61, 62, 65, 70, 74, 86, 90, 90, 95, 101, 107, 114, 114, 124, 128, 133, 136, 141, 145, 150, 150, 157, 161 , 168, 177, 184, 184, 188, 196, 202, 203, 206, 210, 213, 215, 220, 225, 229, 233), macegual (mazahual) (p. 136), metate (p. 136), milpa (p. 230), nahual (pp. 96, 97), nahuite (pp. 116, 129), pajuide (pp. 71, 72, 82, 162, 164, 164, 164, 165, 165, 165, 165, 166, 169, $169,174,177,178,216,234,234,235,235,236)$, petate (pp. 128, 136), tapanco (p. 116), tule (p. 128), zompopero (p. 76).

Doi: https://doi.org/10.15517/rfl.v45i2.39129 / URL: https://revistas.ucr.ac.cr/index.php/filyling/index 
que en el texto se registran con valor toponímico ${ }^{12}$, lo que en principio se puede considerar una prueba indirecta de su presencia, ya en estos momentos, en la variedad salvadoreña/ centroamericana del español. Lo anterior arroja, por tanto, un total de 29 unidades léxicas etimológicamente relacionadas con las lenguas propias del continente ${ }^{13}$.

Ahora bien, una observación más detallada de estos vocablos y -sobre todode su manera de presentarse en el corpus permite detectar la aparición en él de varios ocasionalismos, es decir, "palabras que no pertenecen al uso habitual de la lengua receptora, sino que se usan ocasionalmente en ella (...) con plena conciencia de su condición de extranjeras y sin voluntad de integrarlas" (Álvarez de Miranda, 2009, p. 144). En efecto, mientras que los indigenismos integrados aparecen plenamente incorporados a la narración y carecen de elementos lingüísticos que denoten su carácter de préstamos, los ocasionalismos presentan, por el contrario, una marca discursiva que delata su naturaleza exógena y que muy generalmente adquiere la forma de explicación sobre su significado ${ }^{14}$. Partiendo, pues, de la existencia o no de tal marca lingüística en el texto, es posible clasificar los indigenismos de la Descripción en dos grupos diferenciados (Tabla $1^{15}$ ):

Tabla 1. Indigenismos integrados/no integrados en el corpus

\begin{tabular}{|l|l|l|}
\hline \multicolumn{1}{|c|}{ INTEGRACIÓN } & \multicolumn{1}{|c|}{ CASOS } & \multicolumn{1}{c|}{ VOCES } \\
\hline Voces integradas & 19 & $\begin{array}{l}\text { atole, bejuco, cacahuatal, cacao, cajete, canoa, } \\
\text { ceiba, chicha, chile, chocolate, jacal, jiquilite, maí, } \\
\text { metate, milpa, pajuide, petate, tapanco, tule. }\end{array}$ \\
\hline Voces no integradas & 6 & $\begin{array}{l}\text { calpul, copal, macegual (mazahual), nahual, nahuite, } \\
\text { zompopero. }\end{array}$ \\
\hline TOTAL & $25(100 \%)$ & \\
\hline
\end{tabular}

Como se puede apreciar a partir de estos datos, es claro que la mayor parte de los indigenismos del corpus - un total de 19 voces de 25 , es decir, el $76 \%$ - pertenece al grupo de los integrados ${ }^{16}$, mientras que los ocasionalismos se reducen a seis elementos, cinco

12 Tal naturaleza toponímica resulta indudable, según se ve en sus apariciones: "Este pueblo de Tonacatepeque es la cabecera del curato; tiene [...] un valle llamado Guayabal" (Cortés y Larraz, 2000, p. 214); "a cinco leguas se encuentra la hacienda llamada del Joco" (Cortés y Larraz, 2000, p. 181); "a las orillas de este rio hay hasta veinte y nueve sitios [...]. A la otra banda: 15 El Jocote. 16 Santa Bárbara. 17 El Fresquecito. 18 La Candelaria. 19 La Pitajaya" (Cortés y Larraz, 2000, pp. 177-178).

13 Por supuesto, también aparece en la Descripción (Cortés y Larraz, 2000) abundante onomástica especialmente topónimos (a manera de ejemplo, Nahuisalco, p. 65; Tiotepeque, p. 89; Cuyultitán, p. 123; Chicumquezal, p. 224), pero también hidrónimos y glotónimos (entre otros, Macuilapam, Lempa, p. 218; Comalapa, p. 126; pipil, p. 79; chorti, p. 211; pocomán, p. 235)-de origen indígena; sin embargo, no se incorpora al estudio por sus especiales características, muy diferentes de las que muestran los elementos léxicos ya mencionados.

14 Tal explicación, por supuesto, pretende facilitar al lector la comprensión del término desconocido, y es precisamente ese desconocimiento el que demuestra el carácter no integrado - $\mathrm{O}$, al menos, la interpretación como tal por parte del emisor- del vocablo en cuestión.

15 Como se puede apreciar, se dejan fuera de esta clasificación aquellas voces que aparecen utilizadas como topónimos (guayabal, joco, jocote, pitajaya), habida cuenta de que su empleo como nombres propios impide la aparición de cualquier marca explicativa.

16 De hecho, estos elementos están a veces tan incorporados que incluso son empleados para explicar centroamericanismos semánticos, voces patrimoniales con una especialización significativa

Doi: https://doi.org/10.15517/rfl.v45i2.39129 / URL: https://revistas.ucr.ac.cr/index.php/filyling/index 
nahuatlismos de uso más o menos restringido y uno de los escasos mayismos presentes en el texto ${ }^{17}$. Por lo que se refiere a este último conjunto de voces, resulta interesante analizar también las estrategias discursivas que Cortés y Larraz emplea para incorporarlos en la Descripción geográfico-moral: son fundamentalmente cuatro los métodos que se utilizan con este propósito, a saber, descripción, definición, sinonimia y traducción (Buesa Oliver y Enguita Utrilla, 1992, pp. 41-45) ${ }^{18}$, el autor del texto parece mostrar cierta preferencia por la traducción y la definición, con tres y dos ejemplos de uso respectivamente (copal, nahual y nahuite en el primer caso, macegual y zompopero en el segundo), mientras que la sinonimia y la descripción presentan, cada una, un único ejemplo (calpul, nahuite ${ }^{19}$ ), y son puramente testimoniales (ejemplos a-d):

a. A los animales que se significan en sus sobrenombres, los llaman sus nahuales y quiere decir sus protectores (p. 96).

b. Desdichados de los mazahuales, que es la gente ordinaria, si los indios fueran señores de la América (p. 136).

c. Estos casamientos no se hacen por elección de los muchachos [...], sino de los principales y calpules que mandan con despotismo (p. 83)

d. He notado un supersticioso abuso que llaman nahuite, que se reduce a un obsequio que hacen el cuarto día después de la muerte de cada uno, poniendo en la cama donde murió atoles,

diatópicamente restringida, tal y como demuestra el siguiente ejemplo: "los que se dicen hatos o estanzuelas son algunos jacales que ponen los indios o ladinos en donde se les antoja" (Cortés y Larraz, 2000, p. 152).

17 Con todo, no parece haber una relación clara entre el empleo geográficamente limitado de los indigenismos y la aparición de glosa en la Descripción: a manera de ejemplo, sorprende que el prelado aragonés utilice sin explicación alguna el término pajuide, ausente de los corpus o de los registros lexicográficos, y sin embargo se detenga a definir macegual o copal, ambos ampliamente atestiguados en los repositorios desde el mismo siglo XVI (CORDE, CORDIAM, LEXHISP) y, en el caso de copal, presente ya en el Diccionario de Autoridades (Real Academia Española, 1979, s.v. copal). Es necesario concluir, por tanto, que lo que se está describiendo aquí no es tanto el grado de integración de esas voces en el español centroamericano del siglo XVIII, sino más bien el que presentan -en el marco de los procesos de contacto interdialectal ya mencionados en la nota 8- en el idiolecto de un hablante concreto, en este caso el arzobispo de Guatemala.

18 Siguiendo a estos mismos autores, la descripción se conforma por un segmento textual en que "quedan reflejados los rasgos característicos de los seres y objetos aludidos", mientras que las definiciones facilitan "una fácil y exacta percepción del concepto correspondiente a las voces que las originan" (Buesa Oliver y Enguita Utrilla, 1992, p. 42); por otro lado, la sinonimia supone la configuración de un binomio léxico que implica "una duplicación de vocabulario para la que se utilizan las conjunciones $o$ e y" (Buesa Oliver y Enguita Utrilla, 1992, p. 43) y la traducción, por último, incorpora el indigenismo a través de un verbo de lengua como decir, querer decir o llamar (Enguita Utrilla, 2010, p. 211).

19 Nótese que nahuite es glosado en dos ocasiones y con dos estrategias diferentes.

20 Téngase en cuenta que no todo binomio léxico supone obligatoriamente una glosa: a manera de ejemplo, en el texto aparece con frecuencia haciendas y pajuides (Cortés y Larraz, 2000, pp. 164, 165, etc.), lo que podría considerarse un caso más de sinonimia; sin embargo, la aparición de fragmentos como "el remedio sería quemar los pajuides y que se redujeran a pueblos, y por lo que mira a las haciendas, obligar a sus dueños a que a proporción mantuvieran un eclesiástico" (Cortés y Larraz, 2000, p. 165) o de binomios no sinonímicos al estilo de valles y pajuides (Cortés y Larraz, 2000, p. 166) demuestra que se trata de una simple suma de voces significativamente relacionadas, pero en ningún caso de una glosa explicativa.

Doi: https://doi.org/10.15517/rfl.v45i2.39129 / URL: https://revistas.ucr.ac.cr/index.php/filyling/index 
gallinas, y otras viandas de uso, persuadidos a que viene el difunto ese día a despedirse de su casa y parientes, cuya venida esperan cantando alabados y otras oraciones (p. 129).

Se puede concluir, por tanto, que en la incorporación discursiva de los ocasionalismos el religioso zaragozano echa mano de las mismas estrategias que se descubren desde antiguo en numerosos textos americanos -tales como las crónicas de Indias (Bravo García y Cáceres Lorenzo, 2012, pp. 33-48) o las obras de carácter más o menos científico (Enguita Utrilla, 2010, pp. 207-215; Ramírez Luengo, en prensa c). Asimismo, dentro de las opciones posibles, se decanta especialmente por aquellas que, como la traducción y la definición, permiten integrar de manera efectiva la explicación del indigenismo en el discurso sin necesidad de crear una digresión, algo sin duda conveniente en una obra de carácter narrativo-expositivo como es la que se está analizando.

Desde otro punto de vista, conviene también observar los orígenes etimológicos de los indigenismos que emplea Cortés y Larraz en su texto, por cuanto esta cuestión ayuda a comprender de manera más profunda las lenguas que contribuyen en mayor medida a enriquecer el acervo léxico del español centroamericano. En concreto, a partir de los datos que a este respecto ofrecen diferentes obras lexicográficas -de forma general, Santamaría (1959), el Diccionario Crítico Etimológico Castellano e Hispánico (DCECH, 1980-91), Morínigo (1998), el Diccionario de Americanismos (DAMER, 2010) y el Diccionario de la Lengua Española (DLE, 2014)-, es posible establecer la siguiente distribución de los 29 elementos léxicos resgistrados en el corpus (Tabla $2^{21}$ ):

Tabla 2. Origen etimológico de los indigenismos del corpus

\begin{tabular}{|l|l|l|}
\hline \multicolumn{1}{|c|}{ ORIGEN } & \multicolumn{1}{|c|}{ CASOS } & \multicolumn{1}{|c|}{ VOCES } \\
\hline Náhuatl & $19(65.51 \%)$ & $\begin{array}{l}\text { atole, cacahuatal, cacao, cajete, calpul, chile, } \\
\text { chocolate, copal, jacal, jiquilite, jocote, macegual, } \\
\text { metate, milpa, nahua, nahuite, petate, tapanco, tule. }\end{array}$ \\
\hline Leng. Antillanas & $6(20.68 \%)$ & bejuco, canoa, ceiba, guayabal, maíz, pitajaya.. \\
\hline Maya & $3(10.34 \%)$ & joco, pajuide, zompopero. \\
\hline Cuna & $1(3.44 \%)$ & chicha. \\
\hline TOTAL & $29(100 \%)$ & \\
\hline
\end{tabular}

21 Aunque en general la procedencia lingüística de los términos no resulta dudosa, es importante mencionar la existencia de discrepancias puntuales entre los estudiosos en casos como, por ejemplo, chocolate identificado como náhuatl por la mayoría (DCECH, 1980-1991, s.v. chocolate; Morínigo, 1998, s.v. chocolate; DAMER, 2010, s.v. chocolate) pero de origen maya para Santamaría (1959, s.v. chocolate)o joco, presente como sustantivo únicamente en este último autor (Santamaría, 1959, s.v. joco), quien remite a juco y aporta un étimo maya (juc, macalillo), si bien añade posteriormente "tal vez derivado del azteca xococ, agrio". Como no es el propósito de este trabajo discutir las etimologías de las voces del corpus, se ha aceptado la opinión general de la procedencia náhuatl de chocolate, mientras que en el caso de joco/juco se opta por el maya como fuente del término, siguiendo así la información presentada en primer lugar por Santamaría. También es necesario mencionar el caso de pajuide, ignorado en todas las obras lexicográficas consultadas pero que Hill (2001, p. 150) considera préstamo del kaqchikel pa juyu' 'en la(s) montaña(s)' -vocablo, a su vez, presente con la grafía pajuyúlpajullú en diversas obras salvadoreñas y guatemaltecas de inicios del siglo XIX (Ramírez Luengo, 2017c, p. 68)-, circunstancia que determina su clasificación dentro de los préstamos del maya.

Doi: https://doi.org/10.15517/rfl.v45i2.39129 / URL: https://revistas.ucr.ac.cr/index.php/filyling/index 
Como se puede apreciar, el náhuatl constituye, con 19 voces y el $65 \%$ del total, la lengua que aporta la mayor parte de los indigenismos del corpus, seguido a distancia, con un 20\%, por el arahuaco y el taíno, lenguas que en total aportan seis vocablos. El maya, por su parte, muestra una presencia aún más escasa, reducida a tres elementos léxicos, lo que constituye apenas un $10 \%$ del total, mientras que el cuna, con una sola voz, resulta meramente testimonial. De este modo, el análisis de la Descripción geográfico-moral muestra una situación que no se aparta de lo esperable, tanto en lo que se refiere a la aparición predominante de vocablos nahuas y antillanos como a la escasez de unidades de origen maya, pues tal situación concuerda no solo con lo que tradicionalmente se ha señalado sobre la influencia léxica de las diferentes lenguas del Nuevo Mundo en el español americano -en concreto, con la importancia del náhuatl en los territorios de la Nueva España, con la reducida influencia del maya y con la extensión de las voces antillanas por todo el continente (Ramírez Luengo, 2007, pp. 76-79)-, sino también con los estudios que se han llevado a cabo a este respecto en otras áreas centroamericanas del siglo XVIII como Guatemala (Polo Cano, 2005, pp. 191-192; Ramírez Luengo, Aquino Melchor y Ramírez Vázquez, 2017, p. 115)22.

Más allá de la etimología, tampoco carece de interés el análisis de los campos léxicos a los que se incorpora el indigenismo, pues esta circunstancia permite obtener una idea más precisa acerca de las realidades que de manera predominante son expresadas con préstamos de las lenguas autóctonas. En este sentido, se ha optado una vez más por tomar la clasificación por campos léxicos presente en Quirós García y Ramírez Luengo (2015, p. 197), y los datos que se descubren son los siguientes (Tabla 3):

Tabla 3. Distribución de los indigenismos en campos semánticos

\begin{tabular}{|c|c|c|}
\hline CAMPO LÉXICO & CASOS & VOCES \\
\hline Organización social & $4(13.79 \%)$ & calpul $^{23}$, macegual, nahual, nahuite. \\
\hline Industria / construcción & $4(13.79 \%)$ & bejuco, jacal, pajuide, tapanco ${ }^{24}$. \\
\hline Alimentación & $3(10.34 \%)$ & atole, chicha, chocolate. \\
\hline Flora & $1(3.44 \%)$ & ceiba. \\
\hline
\end{tabular}

22 Tampoco constituye una sorpresa la aparición en el corpus de chicha "bebida alcohólica que resulta de la fermentación del maíz en agua azucarada" (DLE, 2014, s.v. chicha), pues se trata de un vocablo que se documenta desde los inicios del siglo XVI en prácticamente todo el continente (Mejías, 1980, p. 61) y que se mantiene vivo hasta el momento actual en los distintos países de Centroamérica (DAMER, 2010, s.v. chicha).

23 Aunque el DLE (2014, s.v. calpul) define este elemento como "montículo que señala los antiguos pueblos indígenas y que presumiblemente guarda reliquias arqueológicas", el ejemplo de Cortés y Larraz "estos casamientos no se hacen por elección de los muchachos [...], sino de los principales y calpules que mandan con despotismo" (2000, p. 83) demuestra bien a las claras que en este caso su valor es el que facilita Santamaría (1959, s.v. calpul) de "cabeza de grupo o jefe de congregación entre los indios [...], cabeza de familia de la nobleza indígena", presente en la época colonial y habitual, por ejemplo, en la Recordación Florida del guatemalteco Fuentes y Guzmán, país donde aún hoy mantiene un significado cercano, en concreto "persona importante por su autoridad o sabiduría" (DAMER, 2010, s.v. calpul).

24 Una vez más, el ejemplo en el que se presenta el vocablo -"viene el diablo, y haciendo gran ruido en el tapanco de la casa, se despiden" (Cortés y Larraz, 2000, p. 116)- demuestra claramente que su significado para el arzobispo de Guatemala no es el de "puesto, tienda o cajón que se pone en las calles o en los 


\begin{tabular}{|l|l|l|}
\hline Enseres / utensilios & $4(13.79 \%)$ & cajete, copal ${ }^{25}$, metate, petate. \\
\hline Agricultura & $11(37.93 \%)$ & $\begin{array}{l}\text { cacahuatal, cacao, chile, guayabal, } \\
\text { jiquilite, } \text { joco }^{26}, \text { jocote, maiz, milpa, } \\
\text { pitajaya, tule. }\end{array}$ \\
\hline Fauna & $1(3.44 \%)$ & zompopero. \\
\hline Transportes & $1(3.44 \%)$ & canoa. \\
\hline TOTAL & 29 & \\
\hline
\end{tabular}

De este modo, la distribución porcentual de las voces del corpus permite esbozar una situación que, si bien no resulta sorprendente -dada su semejanza con los datos de otros estudios (Ramírez Luengo, Aquino Melchor y Ramírez Vázquez, 2017, p. 117)-, presenta cierto interés para comprender cómo se produce esta estrategia de americanización del léxico, así como las motivaciones que la justifican. A este respecto, quizá una primera cuestión reseñable sea la amplitud de campos léxicos a los que se incorporan estos elementos, así como el hecho de que hagan referencia a ámbitos tan variados como la organización social, la construcción, los utensilios cotidianos y la naturaleza, algo que pone de manifiesto la generalidad que, desde el punto de vista temático, demuestra este proceso y, por consiguiente,

mercados para la venta de comestibles", sino más bien el de "desván, sobrado" (DLE, 2014, s.v. tabanco), es decir, el que la obra académica considera propiamente centroamericano, por más que Morínigo (1998, s.v. tapanco) y Santamaría (1959, s.v. tapanco) lo localicen también en México. En cuanto a la alternancia tapancoltabanco, los diccionarios recogen las dos formas con el mismo significado (con oclusiva sorda en Morínigo, 1998, s.v. tapanco; Richard, 1997, s.v. tapanco; y Santamaría, 1959, s.v. tapanco; y con sonora en el DLE, 2014, s.v. tabanco y el DAMER, 2010, s.v. tabanco) y ambas aparecen desde antiguo en los corpus históricos (CORDIAM, 2016; LEXHISP, 2003), si bien es la variante con sonora la que resulta mayoritaria en Centroamérica, en una situación que parece mantenerse hasta el momento actual en países como Guatemala (DAMER, 2010, s.v. tabanco), Honduras (Herranz, 2017, s.v. tabanco) o el mismo El Salvador (Geoffrey Rivas, 2009, p. 119).

25 Aunque las obras lexicográficas consultadas (DLE, 2014, s.v. copal; DAMER, 2010, s.v. copal; Morínigo, 1998, s.v. copal; Santamaría, 1959, s.v. copal) y los grandes diccionarios del siglo XVIII (Real Academia Española, 1979, s.v. copal; Terreros, 1987, s.v. copal) definen copal como una resina producida por un árbol de la familia de las burseráceas o como el árbol en cuestión, en este caso se ha decidido clasificar este elemento dentro de la categoría enseres/utensilios por el significado de 'vasija, recipiente' con que lo utiliza Cortés y Larraz en su obra, según se puede apreciar en el siguiente ejemplo: "encontró en él ciertos vasos que llaman copales" (2000, p. 76). Cabe preguntarse, a este respecto, si el uso de este indigenismo como 'vaso' constituye un error de interpretación de un vocablo probablemente desconocido para el arzobispo aragonés - nótese que se trata de una de las voces no integradas del corpus- o si, por el contrario, se emplea en el siglo XVIII para referirse a algún tipo de enser doméstico cubierto con los 'barnices duros de buena calidad' que, según el DLE (2014, s.v. copal), se producen a partir deesta resina. A pesar de las dudas existentes al respecto y de la existencia del adjetivo joco 'agrio' (Morínigo, 1998, s.v. joco; Santamaría, 1959, s.v. joco), se ha optado finalmente por incorporar este indigenismo a los vocablos relacionados con la agricultura por dos motivos: en primer lugar, por la aparición de joco y juco como "maíz de grano más grande y más suave que el normal, que se usa para elaborar salpores" en El Salvador (DAMER, 2010, s.v. joco) y "camotillo blanco, de unos cinco centímetros de largo; muy sabrosa legumbre, de carne suave y dulce, algo ácida” en el sur de México (Santamaría, 1959, s.v. juco) respectivamente; en segundo lugar, por la presencia en el texto de diferentes topónimos del actual territorio salvadoreño que responden a nombres indígenas de especies vegetales que son empleadas en la agricultura, tales como El Jocote o La Pitajaya (Cortés y Larraz, 2000, pp. 117-118), paralelos a El Joco si es que se acepta esta interpretación. 
la trascendencia que poseen los indigenismos a la hora de expresar las peculiaridades del Nuevo Mundo. Por otro lado, también es importante mencionar cómo, a pesar de lo que se acaba de señalar, existen algunos campos léxicos en los que la presencia de voces de origen autóctono es especialmente marcada, por ejemplo, las realidades materiales relacionadas con la vida cotidiana -ejemplificadas por los ocho elementos, más del $27 \%$ del total, que engloban la construcción y los enseres/utensilios-, o el mundo de las especies vegetales, que equivalen al $41 \%$ de todos los indigenismos del corpus. Esta situación se justifica precisamente por la originalidad que muestra América en estos aspectos y, por tanto, por la necesidad que tiene el español de dar nombre a unos referentes desconocidos que carecen de denominación en la lengua europea (Ramírez Luengo, 2007, p. 79) 27. $^{27}$

Así mismo, las apariciones en la Descripción de los vocablos que se vienen analizando son también relevantes desde el punto de vista cronológico, ya que -de acuerdo con las búsquedas efectuadas en los grandes repositorios históricos (CORDE, CORDIAM, LEXHISP) y en las obras lexicográficas diacrónicas del español americano (DCECH, DHECR, Company y Melis, 2005; Ramírez Luengo, 2018b)- para un primer grupo de elementos suponen, en ocasiones, sus primeras dataciones absolutas o centroamericanas; para un segundo conjunto de palabras, la única referencia histórica de elementos que carecen de todo registro lexicográfico o que estaban documentados por el momento exclusivamente en la sincronía.

Por lo que se refiere al primer grupo, es importante mencionar el caso del centroamericanismo zompopero "hormiguero de zompopos" (DAMER, 2010, s.v. zompopero), cuya presencia en el español se adelanta algo más de medio siglo -su primera datación hasta ahora se registraba en 1845 en Costa Rica (DHECR, 1995, s.v. zompopo)-, así como jocote "árbol de la familia de las anacardiáceas" (DLE, 2014, s.v. jocote) y metate "piedra sobre la cual se muelen manualmente con el matlapil el maíz y otros granos” (DLE, 2014, s.v. metate), que si bien cuentan con ejemplos en los corpus desde el mismo siglo XVI (CORDIAM, CORDE, LEXHISP), solo ofrecen apariciones centroamericanas de momentos posteriores a la redacción de la obra aquí analizada. En cuanto a los segundos, la ausencia en las fuentes ya mencionadas de joco "maíz de grano más grande y más suave que el normal, que se usa para elaborar salpores" (DAMER, 2010, s.v. joco) y nahuite "levantamiento del espíritu" (DAMER, 2010, s.v. nahuite) transforma los casos registrados en la Descripción en la primera atestiguación histórica de ambos salvadoreñismos, situación en la que también se encuentra el vocablo pajuide "asentamiento pequeño y disperso en la montaña y los barrancos" (Hill, 2001, p. 150). De este modo, el empleo de todas estas voces en el texto de Cortés y Larraz permite constatar su incorporación en el español de la región ya durante la última parte del siglo XVIII, precisión cronológica que avala la profundidad histórica de estos elementos en las variedades centroamericanas de la lengua y que constituye un dato de notable importancia para la más completa reconstrucción de su historia léxica.

27 De hecho, nótese que todos los vocablos del corpus se refieren a elementos poco o nada conocidos en la Península, sean estos realidades culturales (calpul, macegual, nahual, nahuite) o seres y productos propios del continente americano (atole, bejuco, cacahuatal, cacao, cajete, canoa, ceiba, chicha, chile, chocolate, copal, guayabal, jacal, jiquilite, joco, jocote, maí, metate, milpa, pajuide, petate, pitajaya, tapanco, tule, zompopero). Esto explica, a su vez, que la totalidad de ellos sean sustantivos, y que la inmensa mayoría -27 de 29, el 93.10\% del total- sean, además, concretos, de nuevo en coincidencia con lo que se ha descrito ya en otros trabajos sobre la cuestión (Ramírez Luengo, Aquino Melchor y Ramírez Vázquez, 2017, p. 117).

Doi: https://doi.org/10.15517/rfl.v45i2.39129 / URL: https://revistas.ucr.ac.cr/index.php/filyling/index 
Finalmente, conviene también interrogarse sobre la existencia de americanismos dentro de las voces de origen indígena que se están estudiando, por cuanto esta cuestión ayuda a comprender cómo contribuyen tales voces a la creación de la especificidad léxica que parece caracterizar al español centroamericano en general -y salvadoreño específicamente- en el siglo XVIII. Con este propósito, conviene recordar de nuevo que en este caso se define el americanismo a partir de criterios estrictamente basados en el uso (Company, 2007, pp. 28-29), en concreto como aquel elemento léxico que identifica "el habla urbana, popular o culta, o ambas, de América y cuyo uso muy frecuente y cotidiano distancia la variedad americana respecto del español peninsular" (Ramírez Luengo, 2015, p. 116). De tal interpretación derivan, a su vez, dos cuestiones que es fundamental tener en cuenta a la hora de llevar a cabo el estudio histórico de estos vocablos diatópicamente marcados: por un lado, la existencia de tres subtipos de americanismos que Company (2010, p. XVII) denomina puros (voces "empleadas en el español general de América inexistentes en el español peninsular general"), semánticos ("voces y construcciones formalmente compartidas con el español peninsular, pero que han desarrollado en América valores semánticos propios") y de frecuencia ("voces o construcciones compartidas, en forma y significado, con el español peninsular castellano, pero que muestran en América una mucho mayor frecuencia de empleo y de generalización"); por otro -y más importante aún-, el carácter eminentemente dinámico de este concepto, habida cuenta de que la valoración de un vocablo como tal "no se mantiene inalterada a través del tiempo, sino que puede variar a lo largo de la historia, dependiendo de los procesos de expansión o reducción geográfica que experimenten las diferentes unidades léxicas" (Ramírez Luengo, 2012, p. 398) ${ }^{28}$.

Partiendo, pues, de la descripción teórica que se acaba de realizar, lo primero que destaca en los datos del corpus es el hecho de que prácticamente todos los indigenismos se puedan incluir en el siglo XVIII en la categoría de americanismos, en concreto 27 del total, es decir, el 93.10\%. Como excepción a lo anterior se encuentran dos únicos vocablos, chocolate, que se generaliza muy rápidamente por España y que en la Centuria Ilustrada muestra en este territorio proporciones de uso incluso superiores a las americanas, de 16.77 CMP frente a 9.97 CMP, y maíz, que parece transformarse en voz de uso general precisamente en la época en que redacta su Descripción del arzobispo aragonés (Ramírez Luengo, 2017b, p. 110). Por supuesto, esta primera conclusión no resulta en modo alguno sorprendente, pues es de esperar que el hecho -ya mencionado anteriormente- de que los referentes de estos elementos guarden una estrecha relación con las realidades autóctonas americanas determine que los indigenismos presentes en el corpus estén geográficamente circunscritos a las hablas del continente y constituyan, por tanto, una muestra de la dialectalización de este nivel lingüístico ${ }^{29}$. forma de interpretar el americanismo, y uno no menor lo constituye la forma de calcular la frecuencia de uso de los vocablos. En este caso -y a pesar de los problemas de diversa índole que presenta-, se ha optado por utilizar los datos que ofrece CORDE (2016), estableciendo una comparación entre los casos por millón de palabras (CMP) de los fondos identificados como españoles y los que pertenecen a la totalidad de los países de América, única forma de conseguir un volumen suficiente de datos. Para lo que tiene que ver con la problemática que implica el uso del repositorio académico a la hora de llevar a cabo análisis como estos, véase Ramírez Luengo (2017, pp. 614-616).

29 Cabe indicar, con todo, que esto no implica que sean mayoritarios dentro de las voces geográficamente circunscritas que se presentan en el corpus, pues un análisis de todas las unidades léxicas que -siguiendo la metodología ya mencionada- se pueden considerar americanismos en el corpus de Cortés y Larraz 
Junto a lo anterior, la segunda cuestión que se debe analizar en este apartado es el subtipo de americanismos al que pertenecen los 27 indigenismos del corpus que se han identificado como tales en el párrafo anterior; a este respecto, los datos obtenidos son los siguientes (Tabla $4^{30}$ ):

Tabla 4. Americanismos indígenas presentes en el corpus

\begin{tabular}{|c|c|c|}
\hline AMERICANISMO & CASOS & VOCES \\
\hline Puros & $25(92.59 \%)$ & $\begin{array}{l}\text { atole, bejuco, cacahuatal, cajete, calpul, ceiba, chicha, } \\
\text { chile, copal, guayabal, jacal, jiquilite, joco }{ }^{31} \text {, jocote, } \\
\text { macehual, metate, milpa, nahual, nahuite, pajuide, } \\
\text { petate, pitajaya, tapanco, tule, zompopero. }\end{array}$ \\
\hline Semánticos & 0 & \\
\hline De frecuencia & $2(7.40 \%)$ & cacao, canoa. \\
\hline TOTAL & $27(100 \%)$ & \\
\hline
\end{tabular}

Como se puede apreciar, existe una relación muy estrecha entre los indigenismos y los americanismos puros, pues es precisamente a este subtipo al que pertenece más del $90 \%$ del total, algo que se puede relacionar una vez más con el hecho de que, como se dijo anteriormente, estos elementos hagan referencia a realidades propiamente americanas que resultan desconocidas en España y cuya denominación, por consiguiente, se ignora en estas variedades de la lengua. Frente a este claro predominio, el resto de los subtipos ofrecen porcentajes de aparición muy puntuales, pues el americanismo de frecuencia se reduce a dos únicos ejemplos, cacao y canoa, y el semántico ni siquiera se registra ${ }^{32}$, en una situación que no parece ser propia de esta obra, sino que se descubre también en otras del área centroamericana

(2000) demuestra que, mientras que los indigenismos equivalen únicamente al $38.46 \%$ de estos (25 sobre 65), los vocablos endohispánicos superan el 60\%, con 40 elementos del total registrado (Ramírez Luengo, en prensa b).

30 Siguiendo la metodología utilizada en Ramírez Luengo (2017, pp. 610-611), se interpreta como americanismo puro todo elemento que en CORDE (2016) aparece únicamente en textos americanos o bien en aquellos clasificados en este repositorio como españoles pero que presentan temática americana (a manera de ejemplo, la Historia del Nuevo Mundo del jesuita Bernabé Cobo) durante periodo 1701-1800. El americanismo semántico, por su parte, debe presentar en el corpus un significado coincidente con el que existe hoy en día en América y que a su vez difiera del que consta en ciertas obras lexicográficas dieciochescas, muy especialmente el Diccionario de Autoridades (1979). Finalmente, para la detección de los americanismos de frecuencia se utiliza -tal y como se indicó en la nota 28- la frecuencia de empleo del elemento en cuestión por millón de palabras (CMP) en el mismo siglo XVIII, y en concreto se considera como tal aquel elemento que presenta al menos el doble de CMP en América que en España.

31 No se registra ningún ejemplo de este vocablo en la totalidad de CORDE (2016), por lo que se ha optado por considerarlo americanismo puro, dada su nula presencia en España y su aparición en un texto americano como el presente.

32 Si bien es probable que el americanismo puro cacahuatal, que en el corpus presenta el significado de 'sembradío de cacao' (“desde entonces no producen fruto los cacahuatales ni la tierra, y por salir con su antojo, no habrá medio con qué obligarlos a que la cultiven [...], mayormente estando en la inteligencia, como hago memoria de que lo están, de que les agraviaron al Dios del cacao" (Cortés y Larraz, 2000, p. 77), se pueda interpretar en este caso como centroamericanismo semántico, pues, de acuerdo con Santamaría (1959, s.v. cacahuatal), este valor -que considera "intolerable disparate"- resulta propio de toda América Central desde antiguo, dado que lo descubre en la Recordación Florida de Fuentes y Guzmán e incluso es posible detectarlo antes en otros textos guatemaltecos, en concreto desde el mismo siglo XVI (LEXHISP, 2003). 
como es, por ejemplo, la memoria de inicios del siglo XIX del salvadoreño Méndez y Cordero (Ramírez Luengo, 2017c, p. 67).

\section{Conclusiones}

De este modo, el análisis que se ha llevado a cabo a lo largo de estas páginas permite extraer ya una serie de conclusiones acerca del uso de los indigenismos en la sección salvadoreña de la Descripción geográfico-moral que arrojan luz no solo sobre su empleo por parte del autor, sino también sobre la presencia de tales elementos en el español centroamericano de la segunda mitad del siglo XVIII.

En primer lugar, es importante mencionar la aparición en el texto de un conjunto relativamente abundante de estos elementos, en concreto 25 unidades léxicas (atole, bejuco, cacahuatal, cacao, cajete, calpul, canoa, ceiba, chicha, chile, chocolate, copal, jacal, jiquilite, maíz, macegual, metate, milpa, nahual, nahuite, pajuide, petate, tapanco, tule, zompopero) a las que se suman, además, otros cuatros vocablos utilizados como topónimos (guayabal,joco, jocote, pitajaya). Si se tienen en cuenta cuestiones como la naturaleza peninsular de Cortés y Larraz y el carácter de americanismos puros de casi la totalidad de estos términos en el Siglo de las Luces, es posible concluir que muy probablemente se trata de voces que el prelado aragonés ha adquirido a partir de su estancia en Guatemala, lo que no solo demuestra una vez más los efectos que el contacto interdialectal tiene en este nivel lingüístico, sino que además corrobora el empleo de tales voces en el español centroamericano de estos momentos.

Siguiendo con esta cuestión, el análisis de la incorporación discursiva de estos elementos léxicos permite distinguir en ellos dos grupos claramente delimitados: por un lado, aquellos que se pueden considerar integrados; por otro, los ocasionalismos (Álvarez de Miranda, 2009, p. 144), que se caracterizan por contar con una marca discursiva que, en forma de explicación sobre su significado, manifiesta la naturaleza exógena que poseen para el emisor. En el caso concreto del texto analizado, es importante mencionar no solo la presencia de ambos grupos -si bien con una presencia mayoritaria de los primeros-, sino también el empleo, por parte de Cortés y Larraz, de las mismas estrategias discursivas que, a este respecto, aparecen en obras de temática americana de muy distinta naturaleza (Bravo García y Cáceres Lorenzo, 2012, pp. 33-48; Enguita Utrilla, 2010, pp. 207-215), lo que demuestra la utilización, con independencia de la tipología textual, de recursos comunes para enfrentarse al problema compartido de explicar al lector el significado de unos vocablos que muy probablemente desconoce.

En cuanto a los indigenismos en sí, cabe decir que los resultados que se obtienen de su análisis desde distintas perspectivas no resultan demasiado sorprendentes por cuanto coinciden con lo que se ha señalado en otros estudios dedicados a la cuestión: desde el punto de vista etimológico, el náhuatl constituye el origen de la mayoría de ellos (65\% del total), seguido a distancia por las lenguas antillanas y, de manera notablemente más escasa, el maya y el cuna; en cuanto a su distribución por campos léxicos, es destacable tanto la amplitud y variedad de ámbitos referenciales a los que estos elementos se incorporan -en concreto ocho, que van desde la flora hasta la organización social, pasando por los transportes o los enseres y utensilios- como su concentración mayoritaria en algunos de ellos, todo lo cual demuestra la relevancia que posee su empleo a la hora de expresar las realidades más propias del Nuevo Mundo y, en consecuencia, la trascendencia de estos elementos en los procesos que, desde el punto de vista léxico, terminan por configurar el perfil dialectal característico que presentan sus variedades del español. 
En contraste, quizá resulten más interesantes estos resultados desde el punto de vista cronológico, dado que en ocasiones los ejemplos de Cortés y Larraz constituyen la primera aparición, absoluta o centroamericana, de algunos de los indigenismos registrados, o incluso la primera atestiguación histórica de elementos presentes hoy en el español salvadoreño. Por lo que se refiere a los primeros, cabe mencionar el caso de zompopero -datado hasta ahora en el siglo XIX y que, a la luz de los ejemplos del corpus, se utiliza ya en el español en la centuria anterior-, o jocote y metate, para los que, si bien se contaba con casos registrados en México en el siglo XVI (CORDE), no se poseía por el momento ningún testimonio centroamericano, por lo que estas apariciones confirman la profundidad histórica que presenta su uso en la región. En cuanto a los segundos, sin duda destaca el caso de joco y nahuite, recogidos hoy como salvadoreñismos por la lexicografía (DAMER, 2010: s.v. joco, nahuite) y de los que no se poseía hasta el momento ningún registro histórico que pudiera dar cuenta de las fechas probables de su incorporación al español de la región.

Por último, el estudio de los indigenismos del corpus también tiene su importancia para entender la incidencia de estos elementos en los procesos de dialectalización del español centroamericano: en efecto, la aplicación de una perspectiva dinámica y de uso del concepto americanismo (Company, 2007, pp. 28-29; Ramírez Luengo, 2015, p. 116) permite valorar como tales a la inmensa mayoría de los vocablos de origen autóctono utilizados por Cortés y Larraz en su texto, en concreto el $93.10 \%$ del total, y muestra además la estrecha relación que existe entre estas voces y los americanismos puros -subtipo en el que se concentra casi la totalidad de ellas: 25 de 27-, algo que ya se había señalado en momentos previos (Ramírez Luengo, 2017c, p. 67; en prensa b) y que sin duda guarda estrecha relación con el hecho de que tales voces se utilicen mayoritariamente para referirse a realidades propiamente americanas. De este modo, se puede concluir que los datos que ofrece la Descripción geográfico-moral demuestran que para mediados del siglo XVIII se ha producido la incorporación en el español de la región de diversos elementos léxicos de procedencia amerindia que contribuyen a dialectalizar estas variedades del español, es decir, a dotarlas de cierta personalidad que, desde este punto de vista, las distingue de las demás del mundo hispánico (Ramírez Luengo, 2012, p. 395).

En definitiva, no cabe duda de que, por muy diferentes motivos, la Descripción geográfico-moral de Cortés y Larraz se erige en un corpus de notable relevancia para el estudio de la situación léxica que presenta el español de Centroamérica en la segunda mitad del siglo XVIII. Este aserto queda ampliamente demostrado por medio del análisis de los indigenismos que ofrece el texto del prelado aragonés, los cuales constituyen una muestra más que elocuente de los procesos de dialectalización que están afectando ya en estos momentos a las variedades linguiísticas de la región, así como también de la identificación del arzobispo con sus feligreses americanos, cuestión que desborda su misión pastoral y que termina por reflejarse, como se ve a lo largo de estas páginas, en su misma forma de hablar la lengua que comparte con ellos.

\section{Bibliografía}

Academia Mexicana de la Lengua/Academia Nacional de Letras del Uruguay. (2016). CORDIAM. Corpus Diacrónico y Diatópico del Español de América. Recuperado de http://www.cordiam.org

Aguilar y Avilés, G. (2000). Nota introductoria. En P. Cortés y Larraz. Descripción geográficomoral de la diócesis de Goathemala (pp. 9-20). San Salvador: CONCULTURA. 
Álvarez de Miranda, P. (2009). Neología y pérdida léxica. En E. de Miguel (Ed.). Panorama de la lexicología (pp. 133-158). Barcelona: Ariel.

Asociación de Academias de la Lengua Española. (2010). DAMER. Diccionario de Americanismos. Madrid: Santillana.

Bravo García, E. y Cáceres Lorenzo, M. T. (2012). Claves para comprender las Crónicas de Indias. Madrid: McGraw Hill.

Boyd-Bowman, P. (2003). LEXHISP. Léxico hispanoamericano. 1493-1993. Recuperado de http://textred.spanport.lss.wisc.edu

Buesa Oliver, T. y Enguita Utrilla, J. M. (1992). Léxico del español de América: su elemento patrimonial e indigena. Madrid: MAPFRE.

Company, C. (2007). El siglo XVIII y la identidad lingüística de México. México D.F.: Universidad Nacional Autónoma de México - Academia Mexicana de la Lengua.

Company, C. (2010). Introducción. En Academia Mexicana de la Lengua. Diccionario de Mexicanismos (pp. XV-XXIII). México D.F.: Siglo XXI.

Company, C. y Melis, Ch. (2005). Léxico histórico del español de México. México D.F.: Universidad Nacional Autónoma de México

Corominas, J. y Pascual, J. A. (1980-1991). DCECH. Diccionario crítico etimológico castellano e hispánico. Madrid: Gredos.

Cortés y Larraz, P. (2000). Descripción geográfico-moral de la diócesis de Goathemala. San Salvador: CONCULTURA

Enguita Utrilla, J. M. (2010). Indoamericanismos léxicos y estructuras discursivas en la Relación de Cristóbal de Molina. En C. de Molina (Ed.). Relación de las fábulas y ritos de los incas (pp. 199-218). Frankfurt/Madrid: Vervuert/Iberoamericana.

García Añoveros, J. M. (1980). La realidad social de la diócesis de Guatemala a finales del siglo XVIII. Mesoamérica, 1(1), 104-173.

Geoffrey Rivas, P. (2009). La lengua salvadoreña. El español que hablamos en El Salvador. San Salvador: Dirección de Publicaciones e Impresos.

Herranz, A. (2017). Procesos de nahuatlización y nahuatlismos de uso en Honduras. Tegucigalpa: Universidad Nacional Autónoma de Honduras.

Hill, R. M. (2001). Los Kaqchikeles de la época colonial. Adaptaciones de los mayas del altiplano al gobierno español, 1600-1700. Ciudad de Guatemala: Plumsock Mesoamerican Studies / Cholmasaj.

León Fernández, M. (1988). El seseo durante el siglo XVI en Costa Rica. Zona de Cartago (Tesis de licenciatura). Universidad de Costa Rica.

Martín Blasco, J. y García Añoveros, J. M. (1992). El arzobispo de Guatemala Don Pedro Cortés y Larraz (Belchite 1712, Zaragoza 1786): defensor de la justicia y de la verdad. Belchite: Ayuntamiento de Belchite.

Mejías, H. A. (1980). Préstamos de las lenguas indígenas en el español americano del siglo XVII. México D.F.: Universidad Nacional Autónoma de México. 
Morínigo, M. A. (1998). Nuevo Diccionario de Americanismos e Indigenismos. Buenos Aires: Claridad.

Polo Cano, N. (2005). Algunos indigenismos léxicos en el español de Guatemala del siglo XVIII. Res Diachronicae, (4), 185-202. Recuperado de http://resdi.net

Quesada Pacheco, M. A. (1987). El español de Juan Vázquez de Coronado. Análisis descriptivo de un idiolecto de principios de la colonia. Revista de Filología y Lingüistica de la Universidad de Costa Rica, 13(1), 167-182

Quesada Pacheco, M. A. (1995). DHECR. Diccionario histórico del español de Costa Rica. San José: Editorial Universidad Estatal a Distancia.

Quesada Pacheco, M. A. (2009). Historia de la lengua española en Costa Rica. San José: Universidad de Costa Rica.

Quesada Pacheco, M. A. (2013). El sistema verbal del español de Costa Rica en los albores de la época independiente. Signo y Seña, (23), 81-102.

Quirós García, M. y Ramírez Luengo, J. L. (2015). Observaciones sobre el léxico del español del Yucatán (1650-1800). Revista de Filología Española, 95(1), 153-181.

Ramírez Luengo, J. L. (2007). Breve historia del español de América. Madrid: ArcoLibros.

Ramírez Luengo, J. L. (2010). Notas sobre el español salvadoreño del siglo XVIII. En M. T. Encinas et al. (Comp.). Ars Longa. Diez años de AJIHLE (Vol. II) (pp. 879-896). Buenos Aires: Voces del Sur.

Ramírez Luengo, J. L. (2012). El léxico en los procesos de dialectalización del español americano: el caso de la Bolivia andina. Cuadernos del Instituto de Historia de la Lengua Española, (7), 393-404.

Ramírez Luengo, J. L. (2014). Contacto interdialectal en la historia del léxico: los americanismos en la Relación de Aller (Moxos, Bolivia; 1668). En J. M. Santos Rovira (Ed.). Fronteras y diálogos. El español y otras lenguas (pp. 165-176). Lugo: Axac.

Ramírez Luengo, J. L. (2015). El americanismo y la historia: algunas pautas diacrónicas de creación de americanismos (léxicos). Miradas, (13), 114-123.

Ramírez Luengo, J. L. (2017). Aspectos metodológicos para el estudio histórico del léxico americano: conceptos, ejemplificación y tareas para el futuro. Moenia, (23), 603-619.

Ramírez Luengo, J. L. (2017b). Los corpus lingüísticos en la historia del léxico: algunos datos sobre la generalización de los indigenismos antillanos en el español de España. Études Romanes de Brno, 38(2), 101-111.

Ramírez Luengo, J. L. (2017c). El léxico de un culto centroamericano en los inicios del siglo XIX: notas sobre la Memoria del estado político y eclesiástico de la Capitanía General de Guatemala (1821), de J. M. Méndez. Philologica Canariensia, 23(2017), 63-78.

Ramírez Luengo, J. L. (2017d). Textos para la historia del español XI. Honduras y El Salvador. Alcalá de Henares: Universidad de Alcalá. 
Ramírez Luengo, J. L. (2018). Contribución a la historia léxica del español de América Central: algunos americanismos semánticos de la primera mitad del siglo XIX. En E. Carpi y R. M. García Jiménez (Eds.). Herencia e innovación en el español del siglo XIX (pp. 281-294). Pisa: Universidad de Pisa.

Ramírez Luengo, J. L. (2018b). Léxico Histórico del Español de Centroamérica (1650-1819). Honduras. Lugo: Axac.

Ramírez Luengo, J. L. (en prensa). La configuración fónica del español salvadoreño en la época colonial (1650-1803). Boletín de la Real Academia Española.

Ramírez Luengo, J. L. (en prensa b). La Descripción Geográfico-Moral del arzobispo Cortés y Larraz (1770) y la historia léxica de Centroamérica: algunos datos salvadoreños. Cuadernos de Lingüística de El Colegio de México.

Ramírez Luengo, J. L. (en prensa c). Explicar lo desconocido: la incorporación discursiva de los indigenismos en el Florilegio Medicinal de Juan de Esteyneffer. Nueva Revista de Filología Hispánica.

Ramírez Luengo, J. L., Aquino Melchor, S. A. y Ramírez Vázquez, J. J. (2017). Indigenismos léxicos en el español guatemalteco del siglo XVIII. Lengua y habla, (21), 111-125.

Real Academia Española. (1979). Diccionario de Autoridades (edición facsímil de la edición de 1726-1739). Madrid: Gredos.

Real Academia Española. (2014). DLE. Diccionario de la Lengua Española. Madrid: EspasaCalpe.

Real Academia Española. (2016). Banco de datos CORDE. Corpus diacrónico del español. Recuperado de http://www.rae.es.

Richard, R. (1997). Diccionario de Hispanoamericanismos. Madrid: Cátedra

Ruz, M. H. (2002-2004). Memoria eclesial guatemalteca. Visitas pastorales (Vols. I, II, III). México D.F.: Universidad Nacional Autónoma de México.

Santamaría, F. J. (1959). Diccionario de mejicanismos. México D.F.: Porrúa.

Terreros, E. de (1987 [1786-8]). Diccionario castellano con las voces de las ciencias y las artes. Madrid: ArcoLibros.

Ulate Zúñiga, R. (1991). El seseo en el español escrito en el noroeste de Costa Rica durante el siglo XVI. Zonas de Esparza y Guanacaste (Tesis de licenciatura). Universidad de Costa Rica. 
